\title{
Traduire
}

Une autre perspective sur r tr traduction

Revue française de la traduction

$221 \mid 2009$

Voies de l'interprétation

\section{Pratiques et perceptions de la révision en France}

\section{Katell Hernández Morin}

\section{(2) OpenEdition}

Journals

Édition électronique

URL : http://journals.openedition.org/traduire/368

DOI : 10.4000/traduire.368

ISSN : 2272-9992

Éditeur

Société française des traducteurs

Édition imprimée

Date de publication : 15 décembre 2009

Pagination : 58-78

ISSN : 0395-773X

\section{Référence électronique}

Katell Hernández Morin, « Pratiques et perceptions de la révision en France », Traduire [En ligne], 221 | 2009, mis en ligne le 12 novembre 2013, consulté le 25 novembre 2020. URL : http:// journals.openedition.org/traduire/368 ; DOI : https://doi.org/10.4000/traduire.368 


\section{Pratiques et perceptions de la révision en France}

Katell Hernández Morin

\section{Introduction}

Entre le 14 novembre et le 21 décembre 2007, nous avons soumis aux professionnels de la traduction un questionnaire en ligne portant sur la révision et la gestion de la qualité telles qu'ils la percevaient et la pratiquaient (ou non). Cette enquête s'inscrivait dans le cadre d'une thèse de doctorat dont le sujet était : La révision comme clé de la gestion de la qualité des traductions en contexte professionnel.

Le public visé était les traducteurs, réviseurs, chefs de projets ou autres professionnels du secteur de la traduction, inscrits en France. Pour mieux atteindre ce public, un courrier électronique a été envoyé à une liste de diffusion et à quelques associations de professionnels.

Cette enquête nous a paru justifiée dans la mesure où la place de la révision a peu été étudiée, dans la recherche comme chez les professionnels. Les différents observatoires mis en place par les associations de traducteurs étudient la profession dans son ensemble ou par type de structure d'emploi (indépendants, entreprises, etc.), mais ne ciblent pas un type d'activité. L'hétérogénéité de l'offre de traduction en matière de prix, de qualité ou encore de statut du prestataire suggérait également une diversité des comportements en matière de révision. De plus, la révision et la qualité en général étaient au cœur de l'actualité suite à la publication de la norme européenne de traduction NF EN 15038 en 2006 : une norme, rappelons-le, qui impose l'auto-vérification, la révision par un tiers et le contrôle final de la traduction.

Dans ce contexte, nous avons voulu obtenir des données objectives sur les pratiques et les attitudes des prestataires face à la révision, en plaçant celle-ci en situation, dans le cadre de la gestion de la qualité des traductions. Par "gestion de la qualité ", nous entendons l'approche globale de la qualité des prestations et l'ensemble des opérations (y compris la révision) qui en découlent. Contrairement aux autres études empiriques qui visaient essentiellement à mesurer les performances du réviseur, nous cherchions plutôt à : 
- connaître l'ampleur de la pratique de la révision ;

- mieux cerner le rapport des prestataires à la révision ;

- mieux connaître leurs méthodes et leurs habitudes de révision ;

- savoir de quelle autre façon est gérée la qualité de leurs prestations ;

- connaître le niveau de conformité des pratiques de gestion de la qualité avec la norme européenne.

\section{Méthodologie de l'enquête}

Avant d'enquêter sur la révision, le premier problème était de parvenir à une définition satisfaisante du terme lui-même. En effet, la définition n'est pas la même selon que l'on se place du point de vue des chercheurs, des professionnels ou de la norme européenne. Pire encore, plusieurs définitions cohabitent dans la recherche ou le monde professionnel et les appellations utilisées dans la norme européenne restent floues. Une synthèse des réflexions proposées par les chercheurs nous a tout de même permis d'aboutir à la définition suivante :

Révision : examen attentif d'une traduction et mise en conformité de celle-ci avec des exigences de qualité, par le biais de corrections et d'améliorations spécifiques.

Dans notre expérience de la traduction professionnelle et de la révision, la révision correspondait à cette définition, à la différence qu'elle devait nécessairement être effectuée par un autre traducteur ou réviseur que celui qui traduisait. Elle se distinguait ainsi de la relecture, effectuée par le traducteur lui-même.

La norme européenne elle-même envisageait la révision comme réalisée par un tiers, même si les termes de la définition restaient quelque peu confus :

Relecture de révision

Le PST(1) doit s'assurer que le produit de service de traduction a bien été relu pour révision.

Le relecteur-réviseur doit être une personne autre que le traducteur et doit avoir les compétences appropriées dans les langues source et cible. II doit examiner la traduction afin de vérifier son adéquation avec l'objet. Cette tâche doit comprendre, selon les nécessités du projet, la comparaison des textes source et cible afin de s'assurer de l'homogénéité terminologique ainsi que du caractère adéquat du registre et du style.

Afin de permettre aux professionnels interrogés de distinguer la vérification de leurs traductions par leurs soins (relecture ou " auto-vérification ") de l'examen des traductions par un tiers (révision), nous avons retenu ce dernier critère pour la définition de la révision dans l'enquête.

(1) Prestataire de Services de Traduction 
Ce choix terminologique permettait à la fois de parler le même langage que les professionnels (celui d'une partie d'entre eux, tout au moins) et de pouvoir les interroger sur les critères de la norme dans les termes utilisés par celle-ci.

Une fois ces définitions posées, nous avons ciblé plus précisément la population visée. Nous cherchions à atteindre les prestataires de traduction, localisation et révision, qu'ils soient indépendants, salariés de PME, de grandes entreprises ou institutionnels, exerçant notamment en France. Nous voulions obtenir le point de vue des réviseurs comme des "révisés ". Pour accéder à cette large population, nous avons fait le choix d'un questionnaire sur site web, auquel nous avons invité à participer les membres d'un certain nombre d'associations de professionnels. Les associations et la liste de diffusion suivantes ont ainsi été contactées par courrier électronique :

APROTRAD(2), l'UNETICA(3), la CNET(4), la SFT(5), la liste TLSFRM(6), les Traducteurs Experts près la Cour d'Appel de Versailles.

Cette sélection nous permettait de nous adresser à des prestataires au statut et aux spécialisations divers.

Il est difficile d'évaluer la population globale des professionnels de la traduction, tant les statistiques varient ( 6000 à 8000 traducteurs et " assimilés ", voire plus selon certaines sources, dont 5000 à 6000 indépendants). Notre population cible (les membres d'associations) représentait environ 2000 personnes, même si un certain nombre de ces personnes étaient inscrites à deux associations et ont reçu deux fois l'appel à contribution. Nous avons obtenu un total de 115 questionnaires remplis intégralement. Nous les avons dépouillés, puis analysés selon trois méthodes complémentaires : l'analyse univariée ou " tri à plat " des données, l'analyse bivariée par tableaux de contingence et l'analyse multivariée par AFCM (Analyse Factorielle des Correspondances Multiples). Le tri à plat consiste à représenter graphiquement les réponses obtenues pour chaque question de l'enquête. Les tableaux de contingence ou " tableaux croisés " indiquent la distribution des individus interrogés selon deux variables (ou questions) simultanément. L'AFCM, quant à elle, permet de projeter sur un plan géométrique factoriel les individus et les modalités (choix de réponse) d'un certain nombre de variables. Nous ne présenterons dans cet article qu'une partie des résultats de la première analyse, le tri à plat. Pour obtenir les résultats complets des trois analyses, disponibles dans notre thèse, nous renvoyons le lecteur à notre site web(7) ou au site web du serveur de thèses multidisci-

(2) Association PROfessionnelle des métiers de la TRADuction

(3) Union Nationale des Experts Traducteurs Interprètes Près les Cours d'Appel

(4) Chambre Nationale des Entreprises de Traduction

(5) Société Française des Traducteurs

(6) Terminologie et Langages Spécialisés - FoRuM

(7) http://katell.morin.free.fr/ 
plinaire TEL ${ }^{(8)}$. Un article est également en préparation pour présenter les résultats des analyses bivariée et multivariée.

\section{Pratiques et perceptions de la révision}

Notre questionnaire comportait 43 questions, dont les neuf premières permettaient de définir le profil des répondants. La question 1, par exemple, interrogeait les prestataires sur leur structure d'emploi :

Question 1 : Quel prestataire de services de traduction représentez-vous?

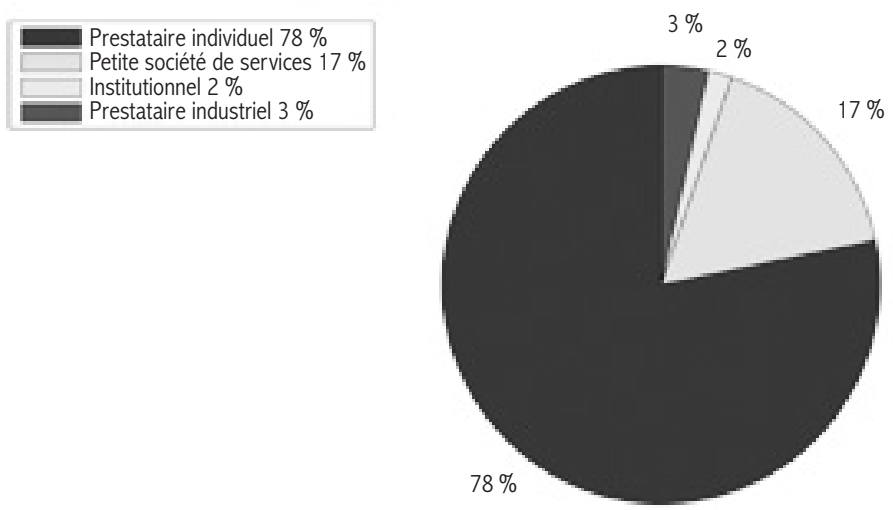

Après dépouillement des résultats - en ce qui concerne la proportion d'indépendants et de salariés notamment, il apparaît que la répartition des personnes sondées correspond aux estimations rassemblées dans un certain nombre de sources : notre question 1 concernant la structure d'emploi des prestataires révèle une proportion de $78 \%$ d'indépendants, pour $17 \%$ de salariés de petites sociétés, $2 \%$ de salariés ou chefs de grandes entreprises et $2 \%$ de fonctionnaires d'organismes publics (soit un total de $22 \%$ de " non-indépendants "). Ces résultats se situent dans les proportions annoncées par Carmelo Cancio dans sa thèse ou par la revue $L^{\prime} E$ tudiant( ${ }^{(9)}$ : entre 70 et $85 \%$ d'indépendants pour la population totale.

La question 2 visait à connaître l'intitulé du poste des répondants :

(8) http://tel.archives-ouvertes.fr/tel-00383266/en/

(9) L'Étudiant. Tous les métiers par secteur. Langues, (page consultée le 18 janvier 2008)

http://www.letudiant.fr/metiers/secteur/langues.html 
Question 2 : Quel est l'intitulé de votre poste?

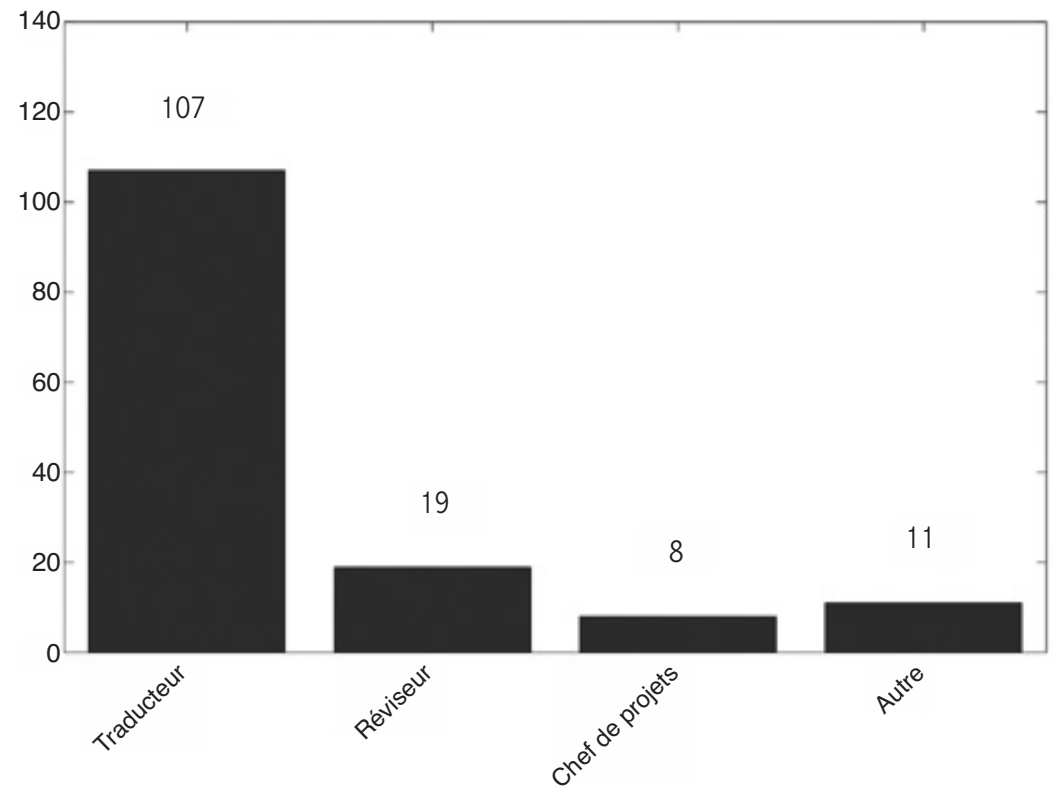

Parmi les intitulés de postes proposés dans notre question 2, nous retrouvons sans surprise une très forte majorité de traducteurs (74\%), qui déclarent ne pas exercer d'autres fonctions. Nous remarquons également qu'une proportion non négligeable des répondants cumule plusieurs fonctions dont celle de traducteur (traducteur et réviseur notamment, dans $8 \%$ des réponses). Le nombre total de personnes ayant déclaré la fonction de réviseur (seule ou en plus d'une ou plusieurs autres fonctions) est de dix-neuf sur 115, alors que le même calcul pour la fonction de chef de projets représente huit personnes. Parmi les autres professions citées (réponses libres), nous retrouvons celles d'interprète, terminologue, gérant de société ou assistant traducteur.

La question 6 du questionnaire permettait d'identifier le type de prestations proposées par les répondants : 
Question 6 : Quel(s) type(s) de prestations vous confie-t-on?

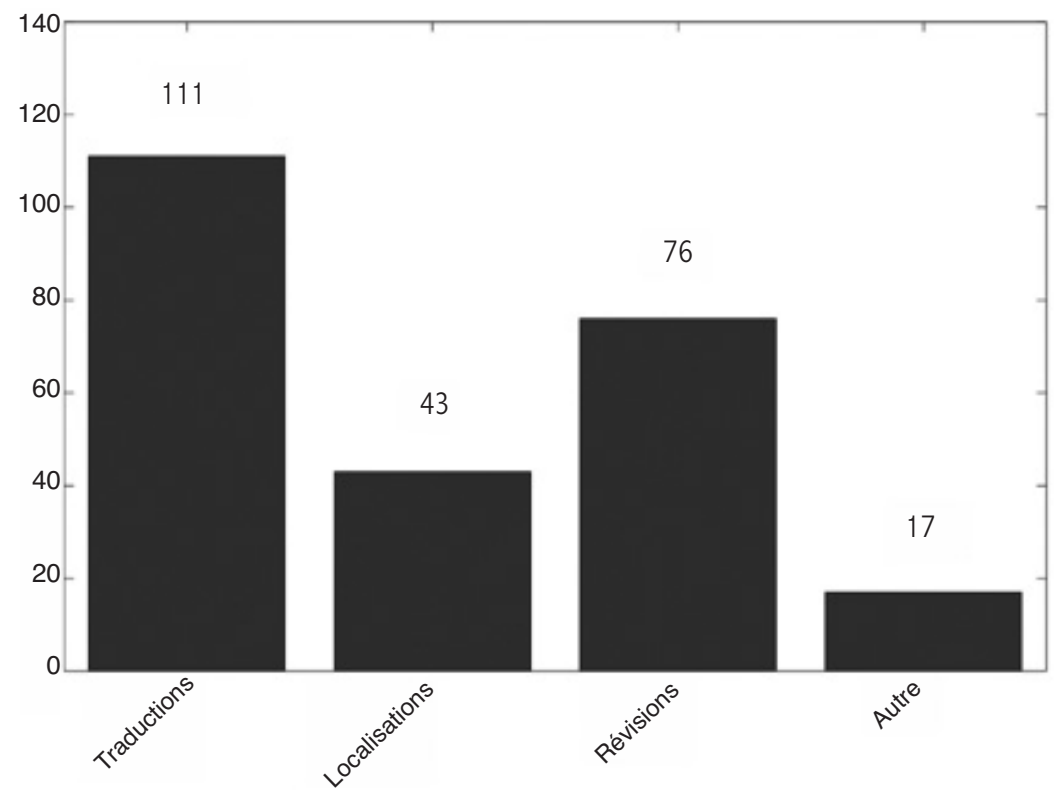

Si la fonction de réviseur est loin d'être majoritaire parmi les personnes interrogées, la révision en tant que prestation proposée est, quant à elle, très présente : $30 \%$ des personnes interrogées déclarent ainsi effectuer des traductions et des révisions. $23 \%$ d'entre elles réalisent à la fois des traductions, des révisions et des localisations. La part totale de la révision dans les réponses fournies s'élève à 76 occurrences, soit plus de la moitié des individus.

Dans la question 5, nous avons demandé aux répondants de préciser le(s) grand(s) domaine(s) ou " genres " de traduction dans lequel (lesquels) ils exerçaient :

Question 5: Dans quel genre de traduction exercez-vous principalement votre activité (technique / littéraire / autre)?

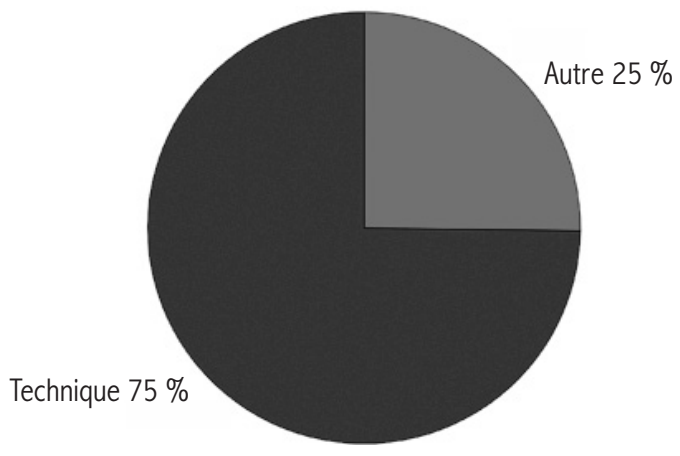




\begin{tabular}{|l|c|}
\hline Juridique & 15 \\
Médical & 4 \\
Financier, économique ou commercial & 8 \\
Publicité, marketing & 1 \\
Rédactionnel & 3 \\
Scientifique & 2 \\
Diplomatique & 1 \\
Jeux vidéo & 1 \\
\hline
\end{tabular}

Réponses libres (modalité "Autre ")

Aucun traducteur littéraire n'a été recensé parmi les répondants. La traduction technique, quant à elle, est très largement représentée, avec $75 \%$ des sondés. Parmi les réponses libres (réponses "Autre "), signalons la part assez importante des professionnels exerçant dans le secteur juridique (quinze réponses), ce qui laisse supposer une bonne participation des membres de I'UNETICA et des Traducteurs experts près la Cour d'Appel de Versailles. Le domaine économique, commercial et financier est également assez bien représenté.

La question 4 avait pour but de connaître le nombre d'années d'expérience des prestataires interrogés :

Question 4 : Depuis combien de temps exercez-vous cette activité ?
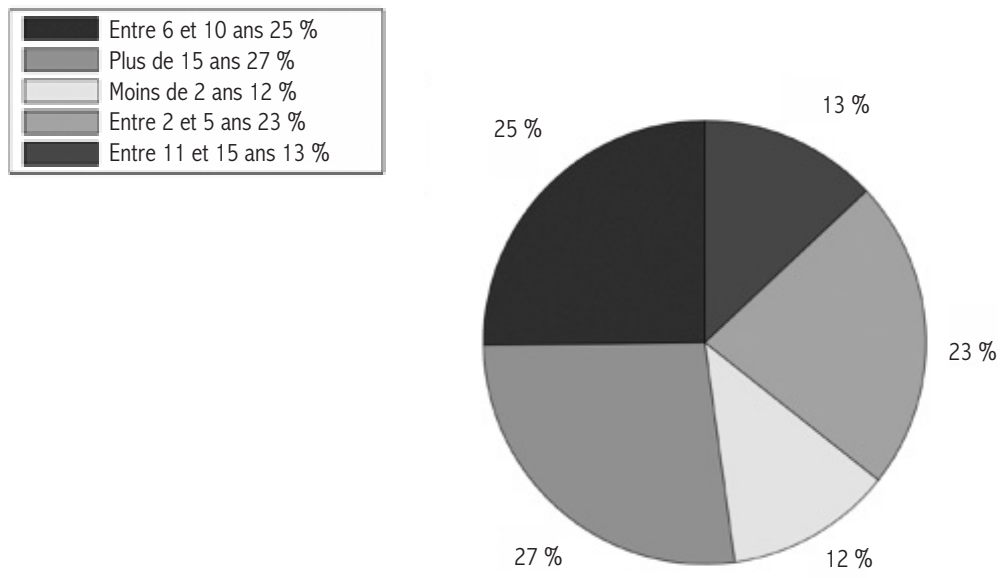
Nous constatons ici une assez grande diversité dans les réponses. Une majorité de participants a plus de cinq ans d'expérience. $27 \%$ des sondés déclarent avoir plus de quinze ans d'expérience et $25 \%$ ont une expérience située entre six et dix ans. $23 \%$ des participants ont entre deux et cinq ans d'expérience.

La dernière question qu'il nous semble important de présenter pour établir un profil des répondants est celle du mode d'exercice de la profession. La question 9 du questionnaire, en effet, nous permettait de savoir si les prestataires travaillaient essentiellement comme soustraitants, pour des clients directs ou des deux façons :

Question 9 : De quelle façon travaillez-vous le plus souvent?

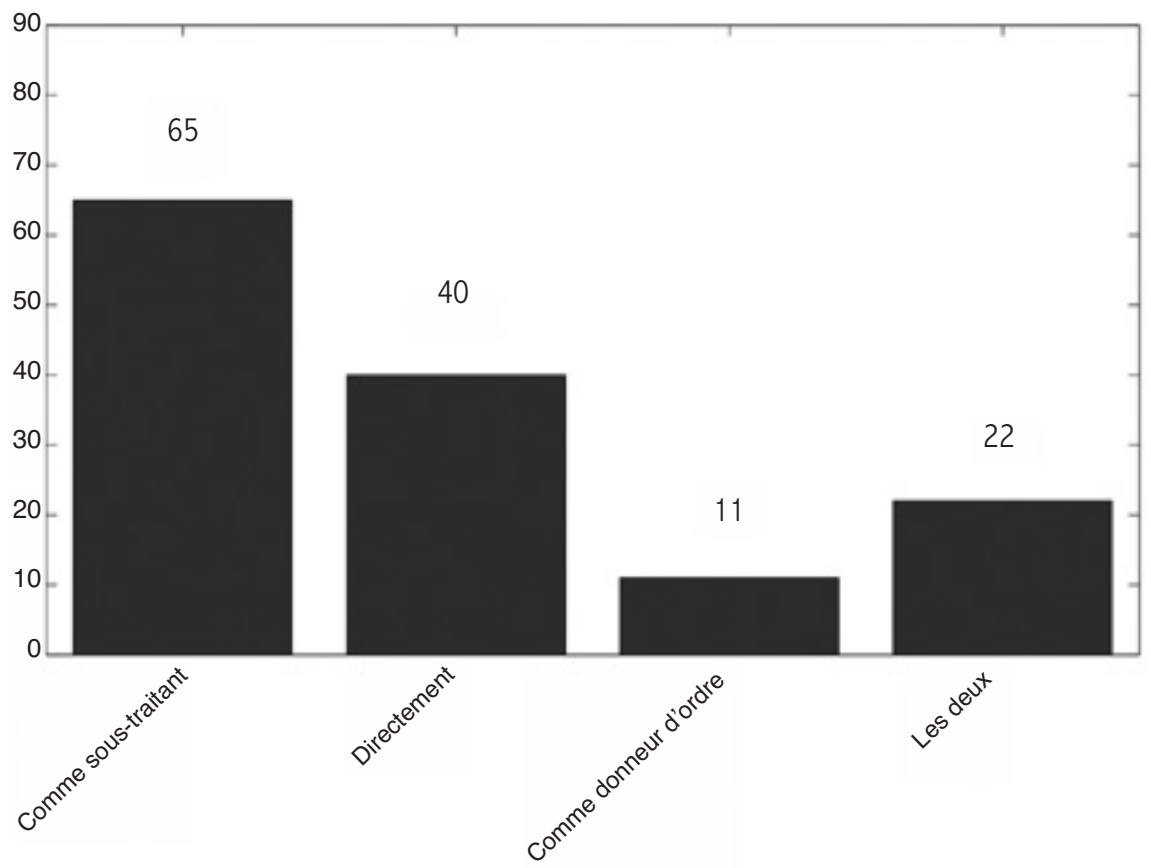

Le mode d'exercice des traducteurs est, le plus souvent, basé sur la sous-traitance : 65 personnes interrogées travaillent en tant que sous-traitants et 22 traducteurs travaillent parfois de cette façon, parfois en relation directe avec leur client. Nous pouvons y voir un rapport avec la répartition de l'échantillon entre indépendants et salariés : les indépendants - majoritaires dans l'échantillon - endossent plus souvent le rôle de sous-traitant que les entreprises. La part des traducteurs agissant comme donneurs d'ordre est limitée dans l'enquête.

Si nous résumons grossièrement le profil moyen des prestataires de l'échantillon, nous pouvons énoncer les conclusions suivantes : 
- il s'agit très majoritairement de traducteurs indépendants ;

- ils effectuent des traductions et des révisions dans des domaines techniques ;

- ils exercent principalement en tant que sous-traitants ;

- le public sondé est plutôt expérimenté.

Les 34 questions de la deuxième partie du questionnaire permettaient d'interroger les prestataires sur leurs pratiques et leurs opinions concernant la révision. Pour cet article, nous retiendrons trois thématiques parmi celles abordées : définition, rôle et besoin de révision, gestion de la qualité, révision et norme.

Comme dans la présentation du profil des répondants, nous avons réordonné les questions pour le bien de la synthèse, même si la structure du questionnaire reste suivie dans les grandes lignes.

Nous reprendrons ainsi notre premier thème, qui rapproche la définition de la révision donnée par les prestataires, le rôle qu'ils lui attribuent et la place qu'elle semble occuper dans les prestations. La première question relevant de ce thème est à mettre en lien avec la question 4 sur l'expérience des répondants:

Question 18 : À partir de combien d'années d'expérience diriez-vous qu'un traducteur est "expérimenté" ?
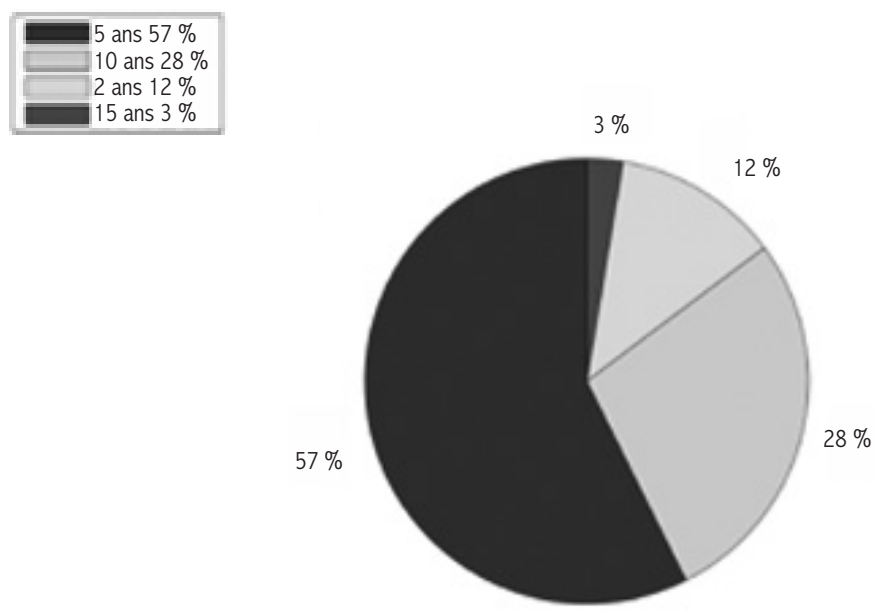

La conception qu'ont les traducteurs de leur " expérience " est exprimée ici en nombre d'années d'exercice - même si la réflexion judicieuse nous a été faite que le nombre d'années ne reflète pas forcément l'expérience réelle du traducteur (exercice à temps partiel ou à temps 
plein, pratique isolée ou diversifiée, etc.). Bien que l'échantillon recueilli soit majoritairement constitué de traducteurs ayant une expérience relativement longue (de six ans à plus de quinze ans d'expérience), il est intéressant de remarquer que ceux-ci considèrent à $57 \%$ qu'un traducteur est expérimenté dès cinq ans d'exercice de la profession.

La question suivante permettait de connaître l'opinion des répondants sur ce qui constitue un " bon " réviseur, en reprenant notamment l'argument de l'expérience :

Question 12 : Parmi les propositions suivantes, laquelle ou lesquelles définit(ssent) selon vous le mieux un bon réviseur?

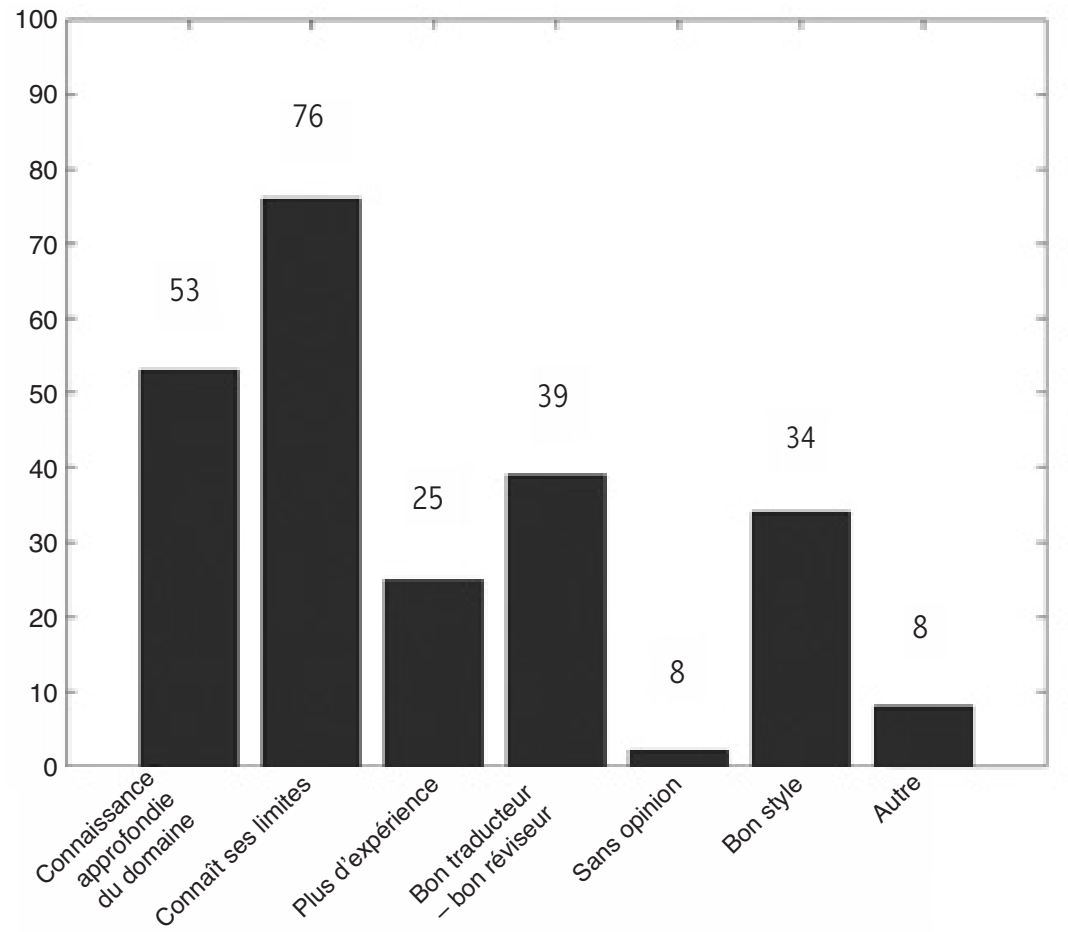

II semble dans cette enquête que la majeure partie des traducteurs interrogés n'envisage pas l'expérience comme un critère prioritaire de définition du " bon " réviseur : 25 personnes sondées seulement affirment que le réviseur doit avoir plus d'expérience que le traducteur ayant effectué la traduction à réviser (soit le cinquième rang parmi les propositions de définition du bon réviseur). Les réponses à cette question sont très variées. Les participants ont combiné, le plus souvent, deux modalités de réponse (deux choix étaient autorisés). Si l'on examine la fréquence de chaque modalité, il apparaît qu'un bon réviseur, selon les personnes 
interrogées, doit avant tout savoir apporter uniquement les modifications nécessaires (76 réponses sur 115) et avoir " une connaissance approfondie du domaine de spécialisation de la traduction ou de la localisation " (53 prestataires).

La question 11, quant à elle, visait à recueillir l'opinion des sondés sur ce qu'apporte la révision à la qualité des traductions :

Question 11 : Selon vous, la révision des traductions ou des localisations apporte beaucoup / peu / rien à leur qualité ?
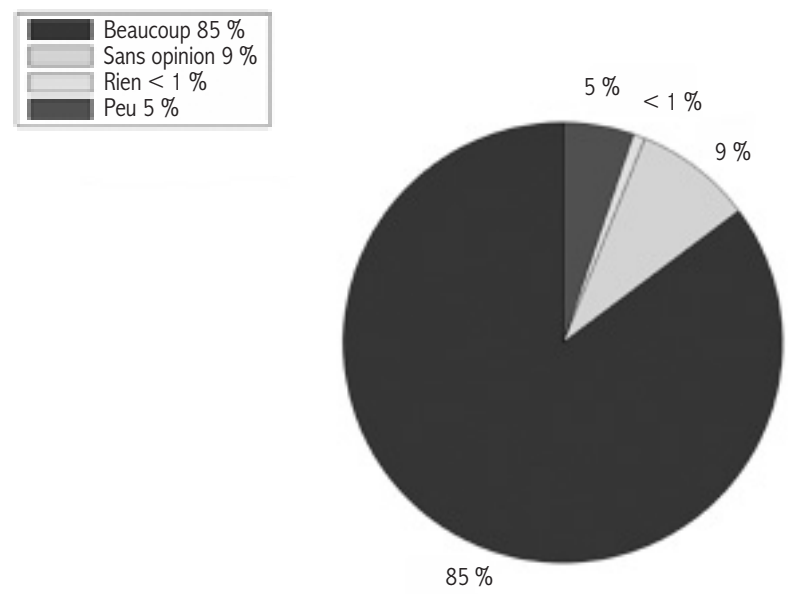

Une forte proportion de répondants a une opinion positive de la révision, si l'on en croit les réponses à cette question : $85 \%$ d'entre eux, en effet, considèrent que la révision apporte beaucoup à la qualité des traductions ou des localisations. Signalons tout de même que $9 \%$ des personnes interrogées déclarent ne pas avoir d'opinion sur le sujet. Peut-être n'ont-elles pas l'expérience de la révision dans leur activité ou estiment-elles que l'apport de la révision est variable selon les situations?

La question 19 complétait la question 11, car elle nous donnait une idée de l'application de la révision comparativement à l'application de la relecture ou "auto-vérification " des traductions : 
Question 19 : En général, combien de relectures et/ou de révisions subissent les traductions ou localisations dont vous avez la charge?
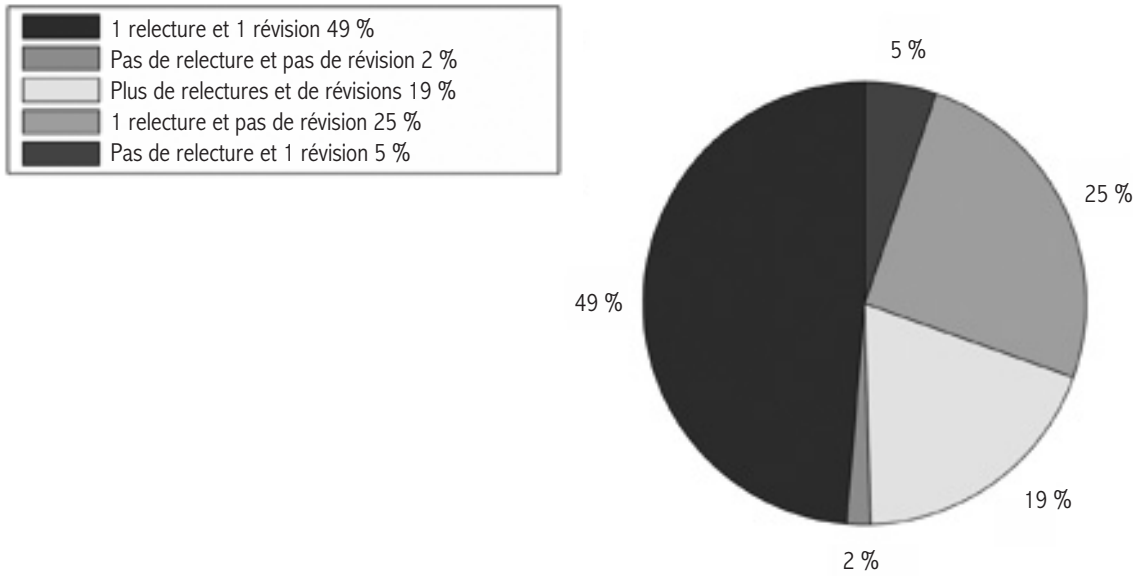

Nous avons souligné que $85 \%$ des traducteurs sondés avaient une opinion positive du rôle de la révision. En ce qui concerne la pratique de la révision elle-même, ils sont en tout $68 \%$ à appliquer au moins une relecture et une révision aux traductions ou localisations qui leur sont confiées. Rappelons que la relecture est ici entendue comme la vérification de sa traduction par le traducteur. La révision est l'examen et l'amélioration de la traduction par une tierce personne (traducteur ou réviseur). Le plus fort taux de réponses revient aux traducteurs disant appliquer " une relecture et une révision ", avec $49 \%$ des sondés. Notons que $25 \%$ des personnes interrogées affirment relire les traductions, mais ne pas réviser (les traductions d'une tierce personne) ou faire réviser leurs traductions. Cette part importante reflète assez bien la pratique de la traduction indépendante (fortement représentée dans l'échantillon), pas toujours facile à concilier avec une révision systématique des traductions par d'autres intervenants.

Les questions suivantes visaient à expliquer pourquoi la révision n'était pas systématiquement utilisée dans la traduction ou la localisation. La question 20, notamment, recueillait les hypothèses des prestataires sur l'omission de la révision : 
Question 20 : Lorsque la révision est omise ou supprimée dans un projet de traduction ou de localisation, pensez-vous que ce soit le plus souvent par :

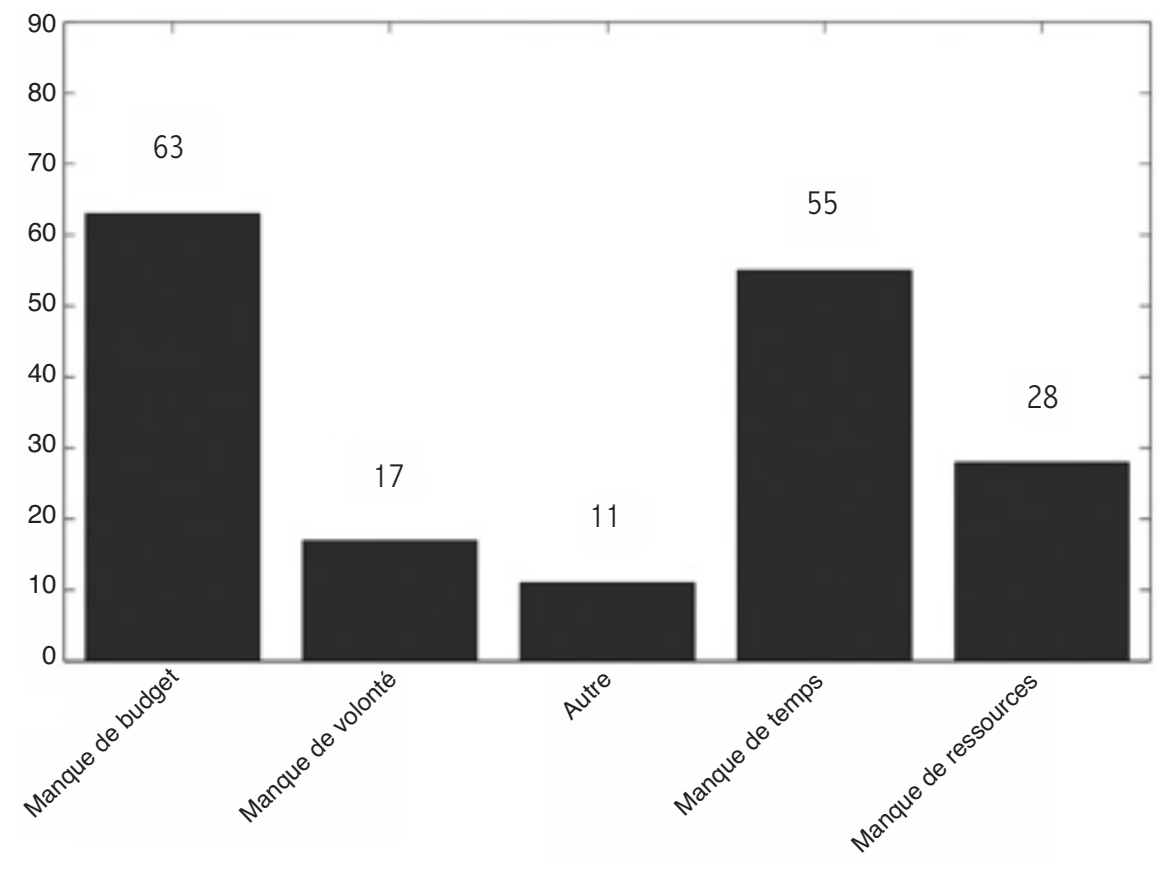

Plusieurs facteurs sont associés dans les réponses, mais les explications revenant le plus fréquemment sont le manque de budget (63 sélections) et le manque de temps (55 réponses).

La question 16 recueillait, cette fois, l'avis des répondants sur la nécessité de réviser systématiquement les traductions : 
Question 16 : Pensez-vous que l'on puisse dans certains cas se passer de révision ?

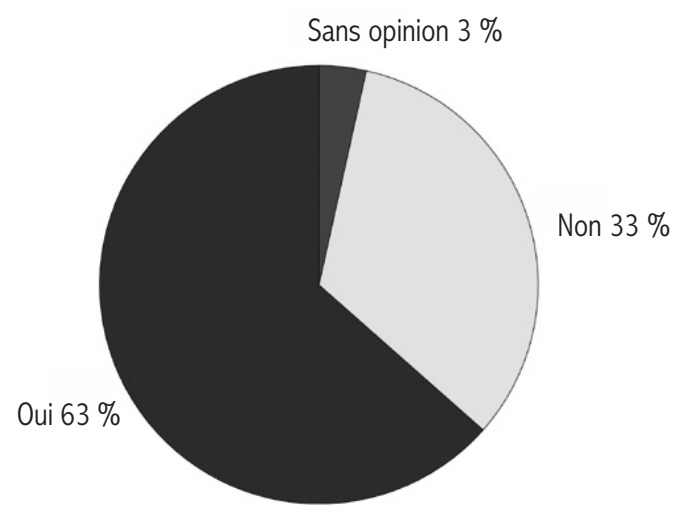

Si la révision ne semble pas toujours possible, elle ne paraît pas non plus toujours nécessaire, si l'on en croit les professionnels sondés : ainsi, $63 \%$ des personnes interrogées estiment que l'on peut dans certains cas se passer de révision.

La question 17 apportait la justification à la question « Pensez-vous que l'on puisse dans certains cas se passer de révision ?":

Question 17 : Si votre réponse est OUl, dans quel(s) cas?

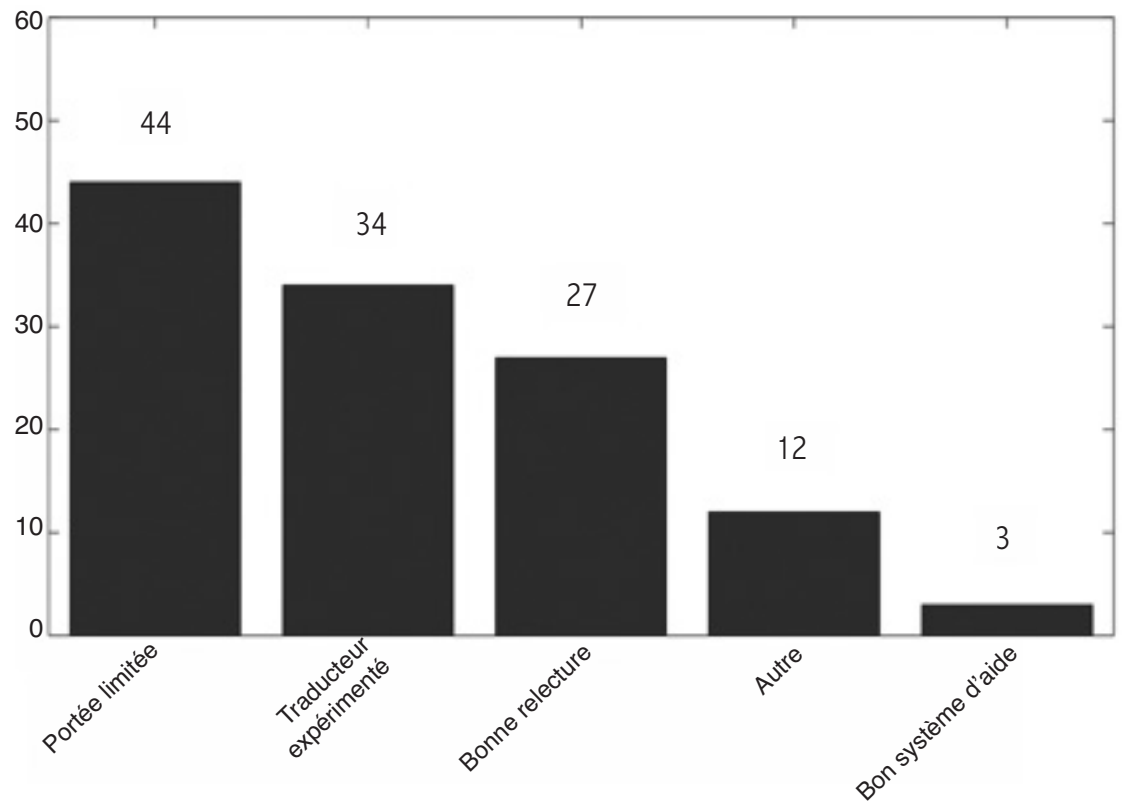


Selon les traducteurs, les cas dans lesquels la révision peut être supprimée sont principalement les suivants : un projet dans lequel la portée du document traduit est limitée (44 sélections); l'expérience du traducteur est jugée suffisante (34) ; ou une bonne relecture est effectuée par le traducteur (27). Ces résultats traduisent la conviction d'une partie de la population selon laquelle le traducteur peut assurer seul la qualité suffisante du matériau traduit. Ils soulignent également la diversité des besoins en matière de qualité.

La dernière question sur le rôle de la révision portait plus précisément sur le niveau d'intervention de la révision sur la traduction :

Question 15 : Selon vous, à quel(s) niveau(x) du discours la révision doit-elle intervenir principalement?

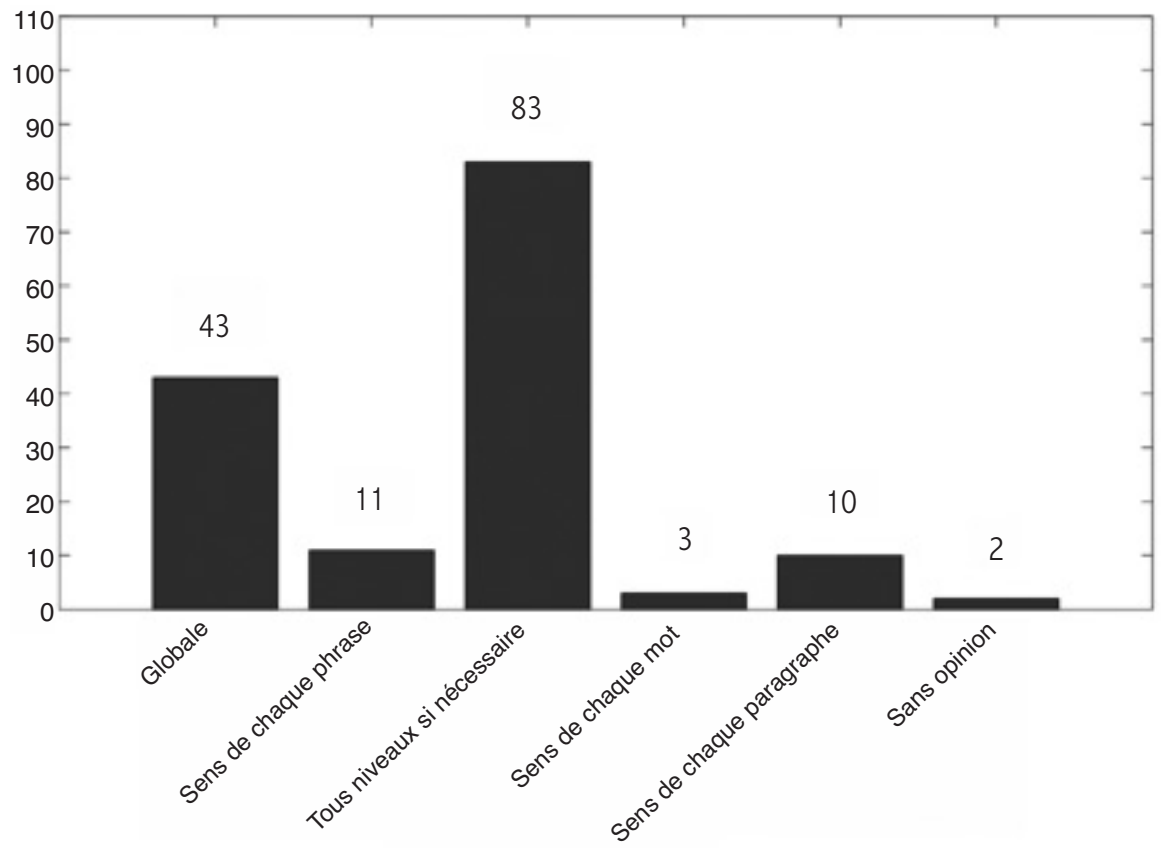

Les avis sont assez tranchés quant aux niveaux de discours sur lesquels la révision devrait intervenir ${ }^{(10)}: 83$ intervenants répondent "à tous les niveaux, si nécessaire " et 43 personnes, " au niveau de la cohérence globale du document ". Les autres propositions recueillent beaucoup moins de sélections.

Cette question du niveau d'intervention de la révision nous renvoie plus globalement à celle de la gestion de la qualité des traductions. La révision n'est qu'une fonction de gestion de la qualité parmi d'autres. Nous avons interrogé les prestataires sur les différentes opérations ou

(10) Examen des mots, des phrases, des paragraphes ou des enchaînements (cohérence globale). 
" procédures " qu'ils appliquaient à leurs traductions dans le cadre de cette gestion de la qualité. Voici ce qu'ils nous ont répondu :

Question 21 : Quelle(s) procédure(s) de gestion de la qualité est (sont) le plus souvent appliquée(s) aux traductions ou localisations dont vous avez la charge?

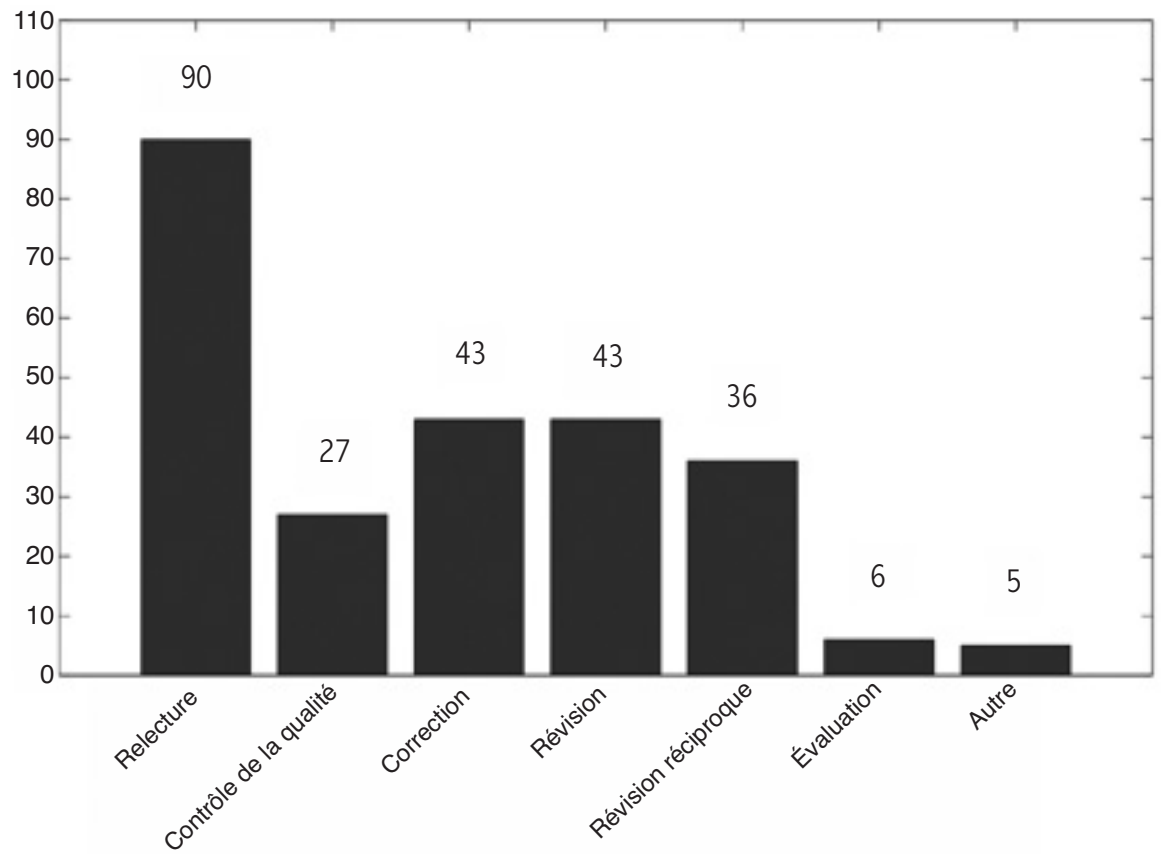

L'omniprésence de la relecture est confirmée par le sondage sur les procédures de qualité : 90 traducteurs déclarent ainsi effectuer une relecture des traductions qu'ils prennent en charge (ou plusieurs, comme nous l'avons vu à la question 19). La révision et la correction (révision sans retour au traducteur) apparaissent ensuite comme les sélections les plus fréquentes, avec la même représentation (43 réponses). La révision réciproque, ou " relecture croisée " entre traducteurs, est également assez souvent appliquée (36 prestataires).

La question 22, complémentaire de la question 21, visait à connaître les autres procédures de gestion de la qualité éventuellement mises en place par les prestataires. Peu de réponses libres ont été apportées à cette question. Six professionnels, néanmoins, ont déclaré effectuer une vérification par " échantillonnage ", spotcheck ou " pointage ".

La confrontation du concept de révision avec celui de contrôle de la qualité nous paraissait également intéressante, surtout dans le cadre de l'explosion de la demande de localisation, particulièrement soumise au contrôle de qualité. La question 23 nous a permis de connaître la perception des répondants à ce sujet : 
Question 23 : Comment différenciez-vous la "révision " du " contrôle de la qualité " ?

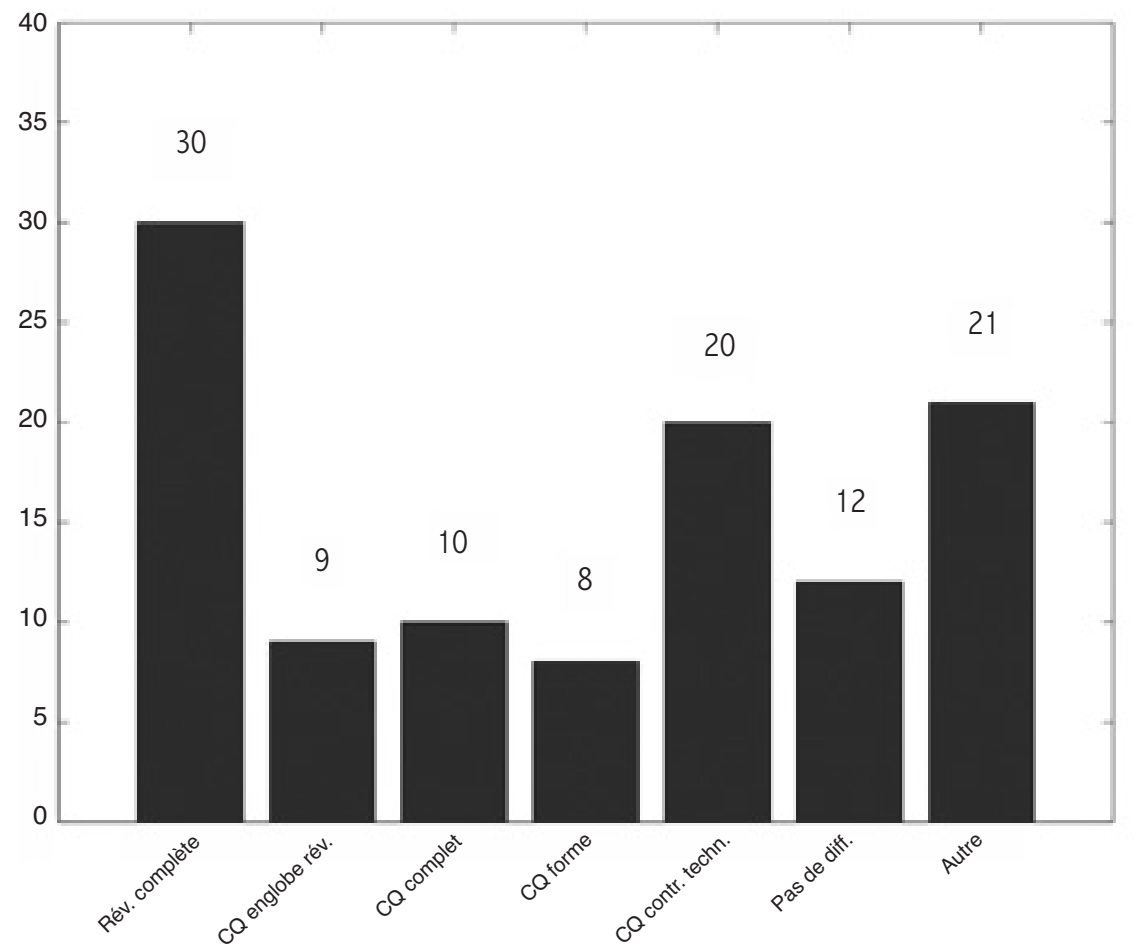

Les réponses à cette question sont très distribuées. Cependant, dans les propositions qui dominent, la révision est définie comme étant plus complète, plus approfondie que le contrôle de la qualité (30 réponses). Le contrôle de la qualité est vu par 20 prestataires comme un contrôle plus " technique ", souvent réalisé par ou pour le client. Douze personnes ne voient aucune différence entre les deux concepts et dix-neuf personnes jugent le contrôle de la qualité plus complet ou plus global, cependant. Certains se réfèrent donc à l'ensemble des contrôles de qualité, quand d'autres voient le contrôle de la qualité comme un contrôle technique ou partiel. Cette question reflète bien la diversité des comportements et le flou qui règne sur les définitions et les usages en matière de qualité dans le monde professionnel.

Enfin, pour être tout à fait en phase avec les problématiques de gestion de la qualité des traductions et de la localisation, nous devions poser la question de la révision des traductions assistées par ordinateur, car les mémoires et autres outils d'aide font largement partie du paysage de la traduction spécialisée aujourd'hui et il est intéressant de savoir comment est assurée la qualité de leur production. La question 40 interrogeait donc les prestataires à ce sujet : 
Question 40 : Quel type de révision appliquez-vous généralement aux traductions assistées par ordinateur dont vous avez la charge?
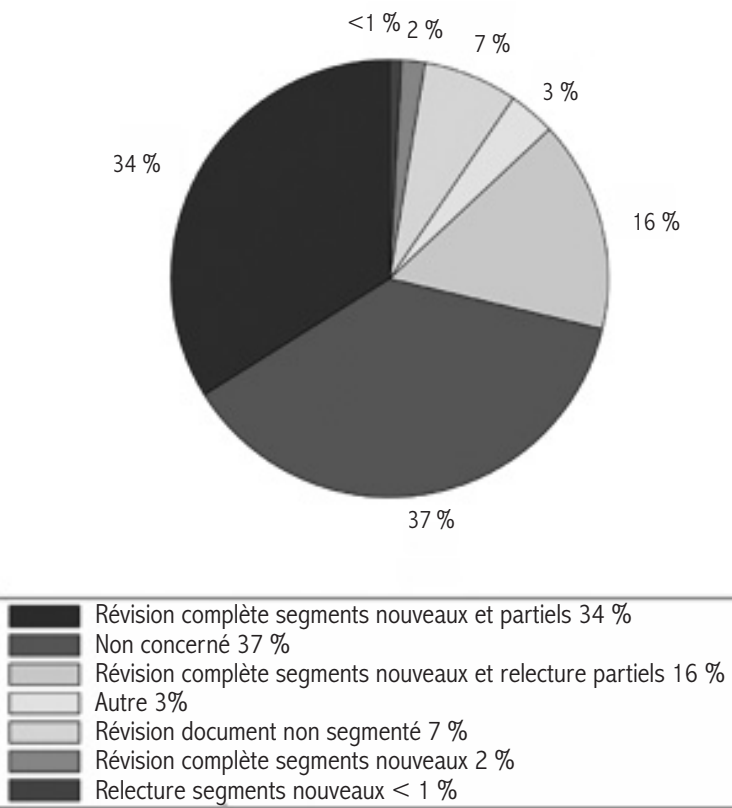

Nous constatons qu'un nombre important de traducteurs interrogés n'utilisent pas les mémoires de traduction (37\% des personnes sondées se disent "non concernées "). Chez ceux qui emploient les mémoires de traduction, nous remarquons une certaine prudence envers les segments partiellement traduits que renvoie la mémoire : ces derniers sont, le plus souvent (34\% des réponses), révisés avec le même soin que les segments nouveaux. $7 \%$ des participants choisissent de restituer tout le contexte du document en le révisant une fois " nettoyé " (sans segmentation). $16 \%$ des traducteurs, en revanche, déclarent effectuer une révision complète des segments nouveaux, mais une relecture rapide des segments partiellement traduits.

Le dernier thème que nous avons souhaité aborder traduit encore l'attitude des prestataires vis-à-vis de la révision et les moyens qu'ils ont de réaliser ce contrôle : il s'agit de l'application de la norme européenne NF EN 15038. La norme impose notamment aux prestataires souhaitant être certifiés une révision de toutes leurs traductions par un traducteur ou un réviseur tiers. Nous avons demandé aux prestataires de l'enquête s'ils souhaitaient et pouvaient appliquer cette norme : 
Question 42 : La norme européenne de traduction NF EN 15038 qui vient d'être publiée indique que toute traduction doit être révisée par une personne autre que son traducteur. Souhaitez-vous appliquer cette norme?

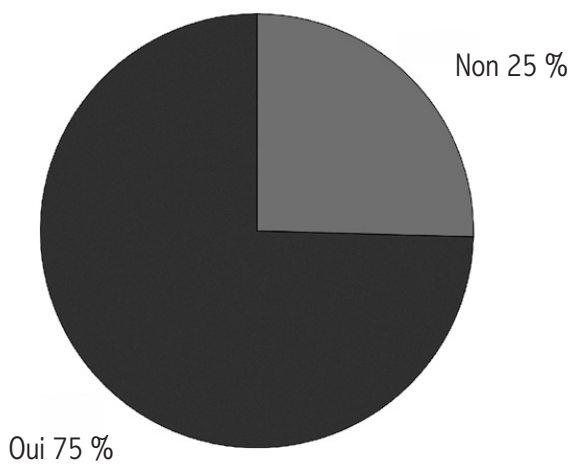

Question 43 : Le pouvez-vous?

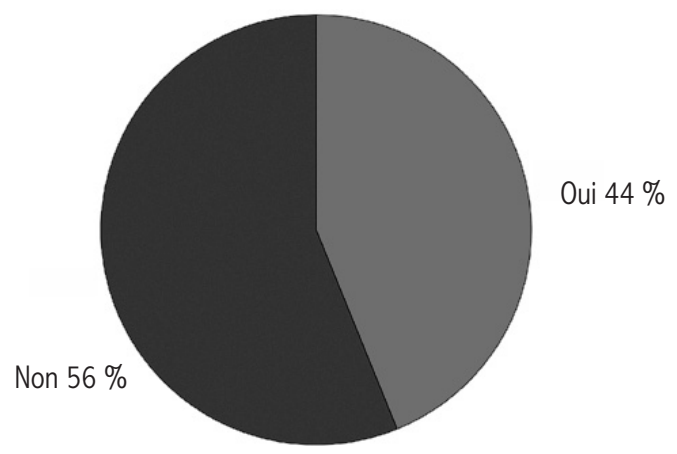

Les réponses à ces deux questions confirment les données obtenues quant à la pratique et à l'omission de la révision dans les projets : $75 \%$ des traducteurs disent souhaiter appliquer la norme, mais $56 \%$ d'entre eux ne le peuvent pas. Selon les répondants, les principales entraves sont les budgets de traduction limités, les délais trop serrés et la difficulté de trouver un réviseur. Autre motif invoqué, la responsabilité de la révision, qui reviendrait au donneur d'ordre et non au sous-traitant (ce que confirme le texte de la norme). 


\section{Conclusions}

Si nous résumons les données apportées par les questions présentées dans cet article, nous pouvons dire que :

- Notre population, très majoritairement constituée de traducteurs indépendants (tout comme la population globale des traducteurs en France, si l'on en croit plusieurs de nos sources), se montre largement favorable à l'application de la révision.

- Au moins un quart de la population de l'échantillon ne pratique jamais la révision.

- Un grand nombre de prestataires semblent s'appuyer sur la relecture pour assurer la qualité de leurs traductions.

- Les motifs de non-révision le plus souvent invoqués sont les prix trop faibles des prestations et les délais de livraison trop serrés.

- Une majorité de sondés juge également que la révision n'est pas toujours indispensable.

- Lorsque la révision est appliquée, elle coexiste avec la correction et d'autres procédures de gestion de la qualité.

- L'attitude par rapport à la norme européenne de traduction est favorable, mais la majorité des prestataires semble ne pas avoir les moyens d'y adhérer totalement.

L'analyse de ces résultats nous donne des indications sur la place importante, mais non prépondérante de la révision dans les pratiques de gestion de la qualité, chez les prestataires interrogés. Nous y voyons également un décalage entre le souhait de travailler d'une certaine façon et la possibilité de le faire. Des facteurs tels que la négociation des contrats, le statut du prestataire, la relation directe ou indirecte entre le traducteur et le client final, l'expérience du traducteur, les différents niveaux de qualité exigés, bref, les conditions générales du marché (ou des marchés), déterminent l'usage de la révision.

Les deux autres analyses (bivariée et multivariée) effectuées au cours de la thèse nous ont permis d'établir certaines corrélations entre ces facteurs et les déclarations des prestataires sondés. Elles confirment ou infirment certaines hypothèses que l'on pouvait, par expérience, formuler sur la pratique de la révision sur le marché français. Le questionnaire reste basé, cependant, sur des déclarations qui ne remplacent pas l'observation directe des pratiques. Les fausses déclarations ou les erreurs de perception sont un risque inhérent à ce genre d'études. Nous savons, néanmoins, que l'observation directe est difficile à mettre en œuvre sur des échantillons importants. Cette contribution permet donc de dresser un panorama global de l'application de la révision. Nous espérons qu'il sera complété par d'autres études et observations plus fines et plus ciblées de la profession. 
Katell Hernández Morin est docteur en Langues étrangères appliquées de l'Université de Rennes 2. Sa thèse a été effectuée sous la direction de Daniel Gouadec et soutenue le 23 janvier 2009. Également titulaire d'un DESS Langues et techniques de l'Université de Rennes 2, elle a cinq années d'expérience en tant que traducteur, réviseur et chef de projets (anglais-espagnol-français) et a exercé la fonction de chargée d'enseignement en LEA, option Traduction, à l'Université de Rennes 2 pendant quatre ans.

\section{Bibliographie}

BRUNETTE, Louise. "Relecture-révision, compétences indispensables du traducteur spécialisé ". Traduction spécialisée : pratiques, théories, formations, 2007, vol. 10, p. 225-235.

CANCIO, Carmelo. La traduction professionnelle en France. Approche du domaine hispanique. 1995. Thèse de doctorat, Université de Toulouse 2.

GOUADEC, Daniel. Qualitrad.net. La qualité en traduction, 2005 (page consultée le 07 juillet 2008), http ://www.qualitrad.net/somm_qual.htm

MARTIN, Tim. "Managing risks and resources : a down-to-earth view of revision ". JosTrans, 2007, vol. 8, (page consultée le 21 juillet 2008),

http://www.jostrans.org/issue08/art_martin.php 